\title{
A PRODUÇÃO DOS BOLSISTAS DE PRODUTIVIDADE DO CNPq EM CIÊNCIA DA INFORMAÇÃO E SUA RELAÇÃO COM AS TEMÁTICAS DO ENANCIB
}

\section{THE PRODUCTION OF CNPq PRODUCTIVITY SCHOLARSHIPS IN INFORMATION SCIENCE AND ITS RELATION TO ENANCIB THEMES}

\author{
Bruno Henrique Alvesa \\ Michely Jabala Mamede Vogel ${ }^{\mathrm{b}}$
}

\begin{abstract}
RESUMO
Introdução: A Sociologia da Ciência busca compreender o comportamento dos pesquisadores e oferecer subsídios para o entendimento de suas práticas de publicação. Ao considerar o Encontro Nacional de Pesquisa em Ciência da Informação (ENANCIB) como o principal evento de Ciência da Informação $(\mathrm{Cl})$ no Brasil, olhar a publicação dos bolsistas de produtividade do Conselho Nacional de Desenvolvimento Científico e Tecnológico (CNPq) à luz dos Grupos de Trabalho (GT) do evento é possível estabelecer um quadro das temáticas da $\mathrm{Cl}$ mais trabalhadas por esses pesquisadores. Objetivo: A Pesquisa buscar compreender a construção do conhecimento científico publicada pelos bolsistas de produtividade do CNPq, agrupando seus artigos de acordo com os GT da Associação Nacional de Pesquisa e Pós-Graduação em Ciência da Informação (ANCIB). Metodologia: Trata-se de pesquisa bibliográfica de natureza quantitativa, qualitativa e exploratória, apoiada em bibliometria. Resultados: Os 51 bolsistas publicaram 883 artigos. Os artigos foram agrupados principalmente nos GT 7, GT 2 e GT 8, e o GT com menos artigos foi o GT10. Identificou-se os pesquisadores mais produtivos por GT bem como suas temáticas e o nível de aderência dos pesquisadores a temática. Conclusões: Destacamos o número de pesquisadores que desenvolvem pesquisas relacionadas as atividades dos GT 2, 7 e 8, por um lado, e a ausência de pesquisadores produtivos nas temáticas relacionadas ao GT 6, que aborda justamente a formação e o perfil esperado nas áreas da Cl. Com o cenário apresentado, arriscaríamos afirmar que os bolsistas $\mathrm{PQ}$ abordam questões relacionadas à organização, recuperação e produção do conhecimento.
\end{abstract}

Descritores: Bolsistas de Produtividade do CNPq. Temáticas de Pesquisa.

a Doutor em Ciência da Informação pela Universidade Estadual Paulista (UNESP). Docente do Departamento de Ciência da Informação da Universidade Federal Fluminense (UFF). E-mail: brhenriquealves@id.uff.br

b Doutora em Ciência da Informação pela Universidade de São Paulo (USP). Docente de Biblioteconomia e Arquivologia e do Programa de Pós-Graduação em Ciência da Informação da Universidade Federal Fluminense (UFF). E-mail: michelyvogel@id.uff.br 
Comunicação Científica. GT da ANCIB.

\section{INTRODUÇÃO}

O estudo das práticas científicas e do fazer científico têm sido alvo de discussões de diversos pesquisadores. Robert Merton, considerado o pioneiro da Sociologia da Ciência, em 1942, já buscava compreender como os cientistas se comportam, o que os motiva, recompensa e intimida. Esse comportamento vem sido medido por meio daquilo que os cientistas entregam à sociedade, que são as publicações científicas, em periódicos, anais de eventos, teses, livros, relatórios, e outros materiais. Nesse sentido, compreender as práticas de publicação pode ajudar a entender o cientista e a própria construção da ciência e de seus campos, tal como proposto por Price, Ziman, Meadows e outros autores.

Uma contribuição para esses estudos é a proposta de Bourdieu, ao abordar o papel do reconhecimento como estímulo ao pesquisador. Esse reconhecimento pode ser mensurado por meio do Capital que "é uma espécie particular de capital simbólico, capital fundado no conhecimento e no reconhecimento" (BOURDIEU, 2008, p. 53). Dessa forma, e observando o cenário brasileiro, questiona-se como os artigos produzidos pelos pesquisadores de produtividade em pesquisa em Ciência da Informação $(\mathrm{Cl})$ contribuem para a construção do conhecimento no Campo em estudo? Como é o comportamento de publicação de nossos pesquisadores?

Como observa Le Coadic (2004, p. 26) a "análise dos processos de construção, comunicação e uso da informação" constitui um dos objetos de investigação da $\mathrm{Cl}$. De práticas de quantificação dos registros do conhecimento visando a administração das unidades de informação, em passado remoto, aos estudos disciplinares de mensuração das atividades científicas, observa-se que os conceitos de Comunicação Científica, e Bibliometria poderiam ser estruturados a partir dos paradigmas da $\mathrm{Cl}$, pois consagram-se como corrente teórica na área (PEREIRA, 2015). Nessa perspectiva, infere-se que a análise da produção científica deveria ser estudada à luz dos contextos informacionais, que 
requerem a compreensão não só do processo de comunicação, mas da própria informação, conceituada por Smit e Barreto (2002, p. 21) como "estrutura simbolicamente significante, codificada de forma socialmente decodificável e registrada e que apresenta competência para gerar conhecimento para o indivíduo e para seu meio".

Nesse contexto, esta pesquisa objetiva, de forma geral, compreender a construção do conhecimento científico divulgada pelos pesquisadores de produtividade em pesquisa em $\mathrm{Cl}$ (bolsistas $\mathrm{PQ}$ ). Esses pesquisadores destacam-se dos demais "[...] já que respondem de maneira expressiva por parte da produção do conhecimento em $\mathrm{Cl}$ no Brasil, além de exercerem influência formativa em seus ambientes de atuação[...]" (CRUZ; SILVA; BUFREM, 2018, p. 4735). De forma específica, propõe-se a agrupar por meio dos procedimentos bibliométricos os artigos publicados pelos pesquisadores de produtividade em pesquisa em Cl, segundo os temas dos Grupos de Trabalho (GT) da Associação Nacional de Pesquisa e Pós-Graduação em $\mathrm{Cl}$, de modo a apontar a esperada coerência entre as temáticas, e observar o comportamento de publicação dos pesquisadores de produtividade em pesquisa em $\mathrm{Cl}$.

A escolha do Encontro Nacional de Pesquisa em $\mathrm{Cl}$ (ENANCIB) se justifica por sua relevância para a área da $\mathrm{Cl}$, uma vez que se trata de "[...] fórum de debates e reflexões que reúne pesquisadores interessados em temas especializados da área, organizados em Grupos de Trabalho" (ANCIB, 2016), considerado evento que promove geração de conhecimento, incentivando a reflexão sobre temas e tendências acadêmicas da atualidade (OLIVEIRA; GRÁCIO, 2009) e o principal evento da frente de pesquisa brasileira em $\mathrm{Cl}$ no Brasil. Seus Grupos de Trabalho (GT) refletem as discussões científicas geradas nos programas de Pós-Graduação, e neste sentido, apresentam as principais temáticas desenvolvidas pela área.

$\mathrm{Na}$ área da $\mathrm{Cl}$ Brasileira, alguns trabalhos foram publicados sobre a produção da produtividade dos bolsistas PQ. Café et al. (2011) e Carvalho et al. (2013) analisaram a produção científica na área da Sociologia, e Melo e Correia (2017) na área da Comunicação. Ferreira, Casarin e Mattos (2016) analisaram as habilidades de busca dos PQ da Educação, enquanto Bufrem et al. (2018) 
buscaram a temática Educação entre as pesquisas dos bolsistas da $\mathrm{Cl}$. Menezes, Oddone e Café (2012) focaram nos aspectos reputacionais dos bolsistas PQ em Cl. Guimarães, Grácio e Matos (2014) analisaram a produção de periódicos dos $\mathrm{PQ}$ em Cl. Castanha e Valentim (2018) investigaram a formação desses bolsistas de Cl, Oliveira, Costa e Oliveira (2018) e Oliveira et al. (2018) investigaram a genealogia dos $P Q$ em Cl, Bufrem, Silva e Sobral (2017), Cruz et al. (2018) e Cruz, Silva e Bufrem (2018) analisaram a influência intelectual desses bolsistas, enquanto Castanha e Grácio (2017) e Melo, Bufrem e Correia (2018) investigaram questões voltadas à autoria. Araújo e Alves (2018, 2019) apresentaram questões da atenção online e altmétrica da produção dos $P Q$. No entanto, o único estudo que buscou aproximar a produção dos $P Q$ em $\mathrm{Cl}$ com a do ENANCIB foi o trabalho de Bufrem, Oliveira e Sobral (2018). Ainda assim, o foco da pesquisa destes autores foram temáticas relacionadas ao GT 7 do ENANCIB.

Nesse sentido, esta pesquisa justifica-se a partir da relevância em compreender o comportamento científico desses pesquisadores por meio da classificação em GT da ANCIB dos artigos por eles publicados nos últimos cinco anos.

\section{COMPORTAMENTO CIENTÍFICO E A COMUNICAÇÃO CIENTÍFICA}

A comunicação científica compreende o conjunto de todas as atividades que englobam a produção, disseminação e uso da informação desde o início do processo de criação científica, desde o princípio onde as ideias da pesquisa são geradas até o momento da aceitação dos resultados como parte do corpo de conhecimento científico (GARVEY, 1979). É a comunicação científica que favorece ao produto (produção científica) e aos produtores (pesquisadores) a necessária visibilidade e possível credibilidade no meio social em que produto e produtores se inserem (TARGINO, 2000, p. 54).

Publicar os resultados científicos é nuclear ao fazer científico. Para Meadows (1999), a comunicação situa-se no coração da ciência. A circulação da informação científica é fundamental para o avanço da ciência, pois é por meio 
da troca de informações que os membros da comunidade científica tomam conhecimento dos resultados das pesquisas realizadas.

Como a informação é o sangue da ciência (LE COADIC, 2004, p. 27), a comunicação científica ocupa um importante papel no reconhecimento científico. Para Vessuri (1987, p. 124), a ciência que não é publicada não existe e para Van Raan (2003, p. 3) todo pesquisador que tem algo importante a dizer, publica. Tais posições corroboram a ideia de Price (1976, p. 4) que afirma que "quando o homem trabalha, produz alguma coisa nova e o resultado é uma publicação, então ele esteve fazendo o que eu chamo de ciência".

Se, a princípio, o ciclo da comunicação científica, conforme proposto por Garvey e Griffith (1979), implicava na realização da pesquisa, apresentação em congresso e discussão com pares, e então publicação em forma de artigo ou livro, atualmente, passou-se a aumentar a quantidade de produtos da mesma pesquisa, especialmente de artigos, não só ao final mas durante diversas etapas da pesquisa. Afinal, como afirma Meadows (1999, p. 85), em termos de comunicação científica, as duas principais características do pesquisador são a quantidade de informações que comunica e a sua qualidade.

De modo a entender o comportamento científico que leva a publicação, Merton (1973) propôs um ethos que norteia a atividade cientifica: Comunismo ou Compartilhamento, Universalidade, Desapego, e Ceticismo sistemático que regem as relações científicas entre pesquisadores (HARGENS, 2004, p. 63). Esses princípios mertonianos ficaram conhecidos pelo acrônimo em inglês CUDOS (Communism, Universalism, Disinterestedness, e Organized Skepticism) e foram acrescidos de um quinto princípio que Ziman (2000) chamou de Originalidade, segundo o qual seria fundamental que as pesquisas contribuíssem com algo novo, seja um novo problema, uma nova metodologia, novos dados, nova teoria ou novas explicações.

Identifica-se que todas essas particularidades indicam a significativa disseminação do conhecimento científico representado pelos diferentes documentos, com o objetivo de atingir um número representativo de usuários e agentes (pesquisadores) (DROESCHER; SILVA, 2014).

De maneira resumida, os princípios de Merton apontam para uma ciência 
livre, aberta, possível de ser verificada, e que foca na atividade científica e não em premiação ou reconhecimento. No entanto, pesquisadores necessitam de infraestrutura para produzir ciência, e passam a concorrer com os demais em buscas de incentivos, bolsas, equipamentos. São, então, submetidos a avaliações, que buscam garantir que o subsídio chegue as mãos daqueles mais capazes.

Darario e Vaccari (2020) defendem que os pesquisadores precisam desenvolver traços de caráter específicos que os capacitem a apreender, produzir e promover os "bens internos" e "externos" das práticas a que se associam. Para os autores, é necessário que um número suficiente de participantes possua plena posse das virtudes, e que disponibilizem os bens internos e externos de forma a educar os demais participantes a seguir seus passos. Uma vez que os pesquisadores apresentam personalidades e experiências diferentes, podem ser capazes de abrigar diferentes grupos de virtudes, mas seria fundamental ao grupo possuir ao menos três diferentes tipos de pesquisador: líder, bom e honesto, cada um dos quais se caracteriza pela posse de certas virtudes.

Ademais, Bourdieu não acredita na existência de atividades científicas puras ou neutras, desvinculadas do político e do social, mas também não vê a ciência sendo apenas determinada por demandas político-econômicas. Para o autor, por seu caráter social, o campo científico tem peculiaridades. Dentre elas, uma certa independência em relação ao mundo global que o envolve (BOURDIEU, 2004, p. 21). Portanto, o campo científico é "um campo de forças e um campo de lutas para conservar ou transformar esse campo de forças" (BOURDIEU, 2004, p. 22-23). Ainda, o campo científico possui uma estrutura que é

[...] definida pela distribuição desigual do capital, ou seja, das armas ou dos trunfos específicos, faz-se sentir, não por interacção directa, intervenção ou manipulação, sobre todos os agentes, mas regulando as possibilidades que thes estão abertas conforme estejam pior ou melhor situados no campo, ou seja, nesta distribuição. O dominante é aquele que ocupa na estrutura uma posição tal que a estrutura age em seu favor (BOURDIEU, 2008, p. 53).

No Brasil, cientistas de destaque podem receber incentivos como, por 
exemplo, as bolsas de Produtividade em Pesquisa (PQ) oferecidas pelo Conselho Nacional de Desenvolvimento Científico e Tecnológico (CNPq). As bolsas PQ são "destinadas aos pesquisadores que se destacam entre seus pares, valorizando sua produção científica segundo critérios normativos [...] e específicos" (CNPq, 2015). São três níveis de bolsa, do mais baixo ao mais elevado: nível 2 (PQ-2), nível 1 (PQ-1), nível sênior (PQ-Sr.), e o nível 1 é, por sua vez, subdividido em outros quatro níveis: 1-D, 1-C, 1-B e 1-A: Dentre tais critérios, destacamos: possuir título de doutor, dedicar-se às atividades constantes do seu pedido de bolsa, produção científica, formação de recursos humanos em nível de pós-graduação.

O comportamento científico dos pesquisadores inseridos em um espaço relacional também esta ligado ao acúmulo dos diferentes capitais simbólicos, tais como, o capital científico do tipo objetivado (produção científica), capital científico do tipo "puro, específico ou autoridade propriamente científica" (prestígio e reconhecimento junto ao campo científico) e capital científico do tipo "institucional, temporal, político ou poder sobre o mundo científico" (ocupação de posições importantes nas instituições cientificas, entre outros). Essas características estão diretamente relacionadas ao papel dos pesquisadores em determinado campo científico, considerando suas singularidades. (BOURDIEU, 2004; BOURDIEU, 2008; ALVES, 2018).

A partir dessa assertiva, entende-se que um dos principais objetivos dos pesquisadores "[...] é buscar aceitação para o que produziram bem como afirmar a sua própria autoridade no papel de produtor científico". Portanto, é necessário definir de forma clara os objetos e problemas a serem investigados, considerando a dinâmica de cada campo científico (DROESCHER; SILVA, 2014, p. 174).

Portanto, é fundamental entender as relações entre pesquisadores, suas temáticas e publicações para se compreender o fazer científico e seus padrões (LEYDESDORFF, 2007). Para tanto, entende-se o campo científico como um sistema social como práticas sociais regulares (GIDDENS, 1989, p. 20). Tal sistema seria um campo de produção científica auto-organizado (WAGNER; LEYDESDORFF, 2005), e a coerência dos autores influência o conteúdo por 
esses desenvolvidos, tal como sugerido por Hanneman e Riddle (2005) e Moody (2004), o que facilitaria a construção de regras e de normas (SCOTT, 2001).

\section{PROCEDIMENTOS METODOLÓGICOS}

Esta pesquisa é de caráter bibliográfico, elaborada a partir da literatura já publicada no tema em questão e de natureza quantitativa, qualitativa e exploratória. Nesse sentido, tem como aspectos investigar o objeto de estudo selecionado inserido em uma realidade multifacetada.

Inicialmente, identificaram-se os pesquisadores de produtividade em pesquisa em $\mathrm{Cl}$, com bolsa em curso no ano de 2019, a partir do site do CNPq, por meio da página eletrônica, nos ícones Plataforma Carlos Chagas, Mapas de investimentos, Brasil, Bolsista de Produtividade em Pesquisa e Tecnologia, Área $-\mathrm{Cl}$.

O corpus de análise foi constituído por 51 pesquisadores de produtividade em pesquisa em $\mathrm{Cl}$, das categorias $1 \mathrm{~A}, 1 \mathrm{~B}, 1 \mathrm{C}, 1 \mathrm{D}$ e 2. Para cada um deles, buscou-se no Currículo Lattes, em dezembro de 2019, a produção científica relativa aos artigos, que resultou em um total de 925 artigos. Nesse sentido, por conta das coautorias, retiraram-se as duplicações, utilizando-se um total de 883 artigos.

Esses artigos foram agrupados com base nos temas dos 11 GTs da ANCIB, a saber:

\section{Quadro 1 - GTs da ANCIB}

GT 1: Estudos Históricos e Epistemológicos da Ciência da Informação - compreende os seguintes temas: Construção do conhecimento na área e questões epistemológicas, interdisciplinaridade, transdisciplinaridade, entre outros.

GT 2: Organização e Representação do Conhecimento - aborda as seguintes temáticas: teorias, metodologias, processos, produtos, preservação da memória institucional, instrumento para a representação do conhecimento, entre outros.

GT 3: Mediação, Circulação e Apropriação da Informação - apresenta os seguintes temas: construção do campo da $\mathrm{Cl}$, contribuições teórico-metodológicas diversificadas em sua constituição, entre outros.

GT 4: Gestão da Informação e do Conhecimento - compreende os seguintes temas: gestão de ambientes, unidades de informação, produtos, serviços, recursos organizacionais, instrumentos de gestão, aprendizagem organizacional, entre outros. 
GT 5: Política e Economia da Informação - destaca as seguintes temáticas: regimes de informação, acesso à informação, ética da informação, informação e ecologia, entre outros.

GT 6: Informação, Educação e Trabalho - aborda os seguintes temas: competências e habilidades, sociedade do conhecimento, formação profissional, modelos curriculares, entre outros.

GT 7: Produção e Comunicação da Informação em CT\&I - compreende os seguintes temas: estudos teóricos e metodológicos, avaliação e gestão em CT\&l, entre outros.

GT 8: Informação e Tecnologia - apresenta as seguintes temáticas: geração, armazenamento, recuperação, disseminação da informação, preservação da informação, ambientes digitais, entre outros.

GT 9: Museu, Patrimônio e Informação - destaca os seguintes temas: perspectivas teóricas e práticas de análise, aspectos informacionais e comunicacionais, entre outros.

GT 10: Informação e Memória - aborda os seguintes temas: representações sociais e conhecimento, virtualização da memória social, coleções e colecionismo, entre outros.

GT 11: Informação \& Saúde - aborda os seguintes temas: tecnologias em inovação em saúde, capacitação em informação em saúde, entre outros.

Fonte: Enancib (2019).

Utilizaram-se o título, resumo e palavras-chave para categorizar os artigos em um ou mais GT (não mutuamente exclusivos, ou seja, o artigo poderia estar relacionado em mais de um GT da ANCIB). Com isso, os pesquisadores de produtividade em pesquisa do CNPq foram associados a um dos GT da ANCIB por meio dos artigos que produziram (ALVES, 2018).

Ao realizar o agrupamento dos artigos ocorreram algumas dificuldades, tais como, a falta de especificidade, principalmente, no resumo apresentado pelo artigo, empregando algumas vezes termos genéricos no contexto social e científico da $\mathrm{Cl}$.

Ainda, para gerar a rede two-mode (Grafo 1) entre as duas variáveis (pesquisadores e GTs) utilizou-se o software Ucinet a partir da matriz 51X11.

\section{APRESENTAÇÃO E ANÁLISE DOS DADOS}

A Tabela 1 apresenta a categoria/nível dos pesquisadores de produtividade em pesquisa em $\mathrm{Cl}$; número de bolsistas; número de artigos; média de artigos e desvio padrão, no período em estudo.

Esses pesquisadores publicaram um total de 883 artigos. Nesse sentido, observa-se na Tabela 1 , que somente dois pesquisadores $(3,9 \%)$ pertencem à 
categoria/nível $1 \mathrm{~A}$ e publicaram uma média de 6,5 artigos cada um, com um desvio padrão igual a 3,5. O nível A "é reservado a candidatos que tinham mostrado excelência continuada na produção científica e na formação de recursos humanos, e que liderem grupos de pesquisa consolidados" (CNPq, 2015).

Tabela 1 - Categoria/nível dos pesquisadores de produtividade em pesquisa em $\mathrm{Cl}$, número de bolsistas, número de artigos, média de artigos e desvio padrão

\begin{tabular}{ccccc}
\hline Categoria/nível & $\begin{array}{c}\text { Número de } \\
\text { bolsistas }\end{array}$ & $\begin{array}{c}\text { Número de } \\
\text { artigos }\end{array}$ & $\begin{array}{c}\text { Média de } \\
\text { artigos }\end{array}$ & $\begin{array}{c}\text { Desvio } \\
\text { Padrão }\end{array}$ \\
\hline 1A & 2 & 13 & 6,5 & 3,5 \\
1B & 5 & 109 & 21,8 & 11,7 \\
1C & 2 & 12 & 6,0 & 4,2 \\
1D & 9 & 132 & 14,7 & 14,1 \\
2 & 33 & 659 & 20,0 & 9,8 \\
\hline
\end{tabular}

Fonte: Elaborado pelos autores.

Foram identificados cinco pesquisadores $(9,8 \%)$ na categoria $1 \mathrm{~B}$, que publicaram uma média de 21,8 artigos cada um, com um desvio padrão igual a 11,7 ; e dois pesquisadores (3,9\%) que são $1 \mathrm{C}$ e produziram uma média de 6,0 artigos cada um, com um desvio padrão igual a 4,2. Nesse contexto, espera-se que esses pesquisadores "participem de forma significativa em atividades de pesquisa em suas instituições e, no caso dos pesquisadores $B$, em órgãos de fomento à pesquisa" (CNPq, 2015). Ainda, observa-se nove pesquisadores $(17,6 \%)$ que são 1D e publicaram uma média de 14,7 artigos cada um, com um desvio padrão igual a 14,1. Com isso, é importante que esses pesquisadores enquadrados na categoria 1 tenham uma significativa visibilidade científica em âmbito nacional e internacional.

Observa-se 33 pesquisadores $(64,7 \%)$ na categoria 2, que produziram uma média de 20,0 artigos cada um, com um desvio padrão igual a 9,8. Para essa categoria "será avaliada a produtividade do pesquisador, com ênfase nos trabalhos publicados e orientações, ambos referentes aos últimos 5 (cinco) anos". (CNPq, 2015).

Por fim, destacam-se os diferentes números de pesquisadores em diversas categorias/níveis, com maior frequência de pesquisadores bolsistas PQ em Cl na categoria/nível 2. 
A maior média de artigos produzidos ocorre na categoria/nível 1B, com um desvio padrão de 11,7. O maior desvio padrão é apresentado pelos pesquisadores categoria/nível 1D com 14,1.

Apresenta-se, no Grafo 1, a rede two-mode, na qual se relacionam os 51 pesquisadores de produtividade em pesquisa em $\mathrm{Cl}$, representados por círculos vermelhos, e os GT da ANCIB, representados por quadriláteros azuis. A espessura dos segmentos que unem o pesquisador ao respectivo GT corresponde à frequência com que ele produziu na temática. Os três GT que apresentaram o maior número de artigos foram:

O GT 7 - "Produção e Comunicação da Informação em Ciência, Tecnologia \& Inovação" - compreende as seguintes temáticas: estudos teóricos e metodológicos; avaliação da produção científica, entre outros (ANCIB, 2016). Nesse GT foram categorizados 263 artigos, sendo 28 (54,9\%) pesquisadores responsáveis por eles, com destaque para Maria C. C. Grácio, com 35 artigos; Maria C. P. I. Hayashi, com 32 artigos; Sámile A. de S. Vanz, com 29 artigos; Adilson L. Pinto, com 25 artigos.

O GT 2 que se refere à "Organização e Representação do Conhecimento", e aborda os seguintes temas: organização e preservação de documentos; ambientes informacionais; representação do conhecimento, entre outros (ANCIB, 2016). Foram sistematizados 253 artigos com foco nesse GT, considerando o total de 51 pesquisadores, sendo $34(66,7 \%)$ os responsáveis por artigos nesse tema. Destacam-se os pesquisadores com maior número de artigos nesse GT da ANCIB: Mariângela S. L. Fujita, com 32 artigos; José A. C. Guimarães, com 30 artigos; Gercina Â. de Lima, com 24 artigos; Fábio A. Pinho, com 22 artigos.

No GT 8 - Informação e Tecnologia - compreende os seguintes temas: representação, recuperação, disseminação da informação, entre outros (ANCIB, 2016). Foram encontrados 165 artigos enquadráveis nesse GT da ANCIB, sendo 26 pesquisadores responsáveis por eles, com destaque para Silvana A. B. G. Vidotti, com 33 artigos; Guilherme A. Dias, com 16 artigos e Silvana D. Monteiro, com 12 artigos. 


\section{Grafo 1 - Rede two-mode formada pelos pesquisadores de produtividade em pesquisa em $\mathrm{Cl}$ e GTs da ANCIB.}

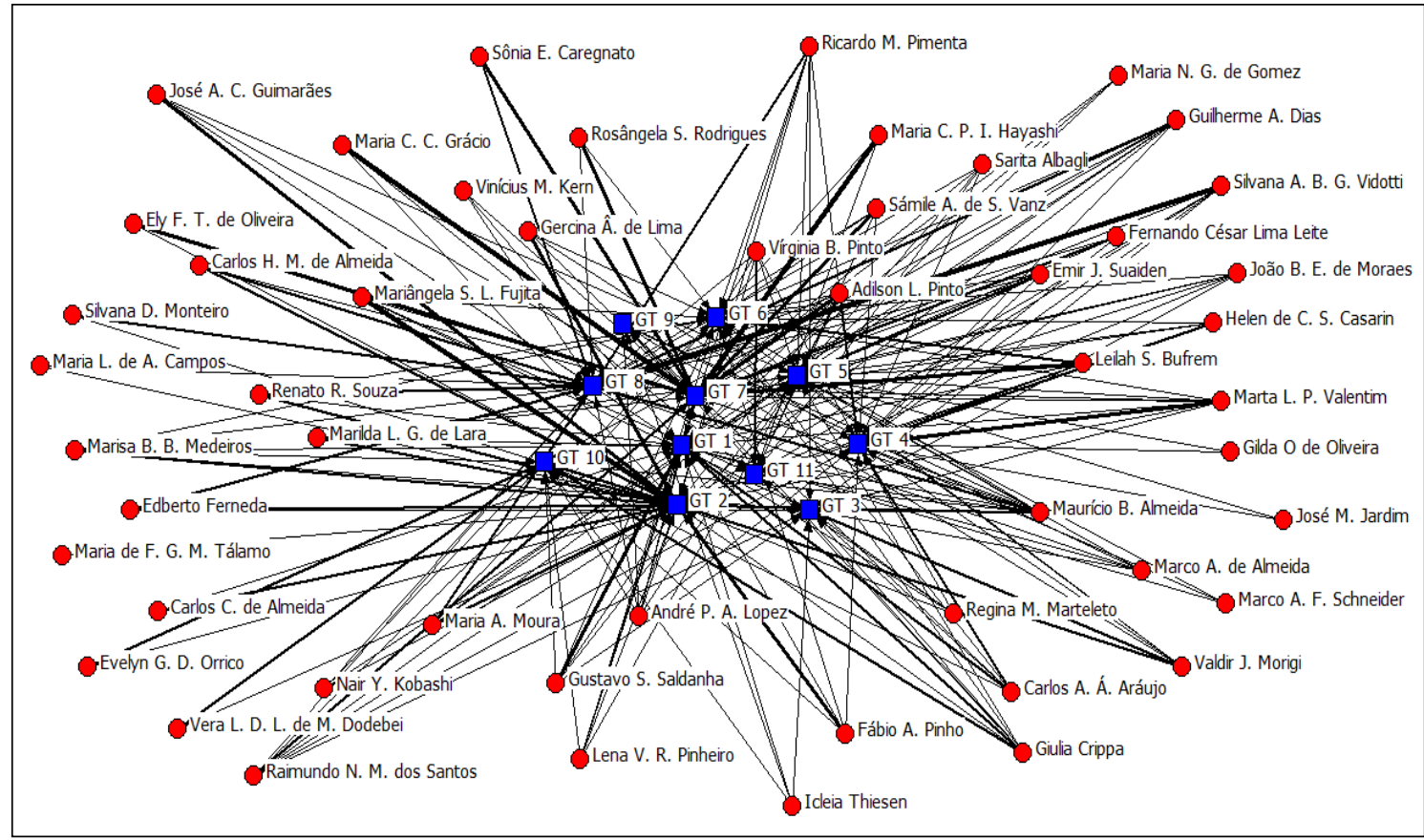

Fonte: Elaborado pelos autores por meio do Software Ucinet.

Por fim, o GT 1 - Estudos Históricos e Epistemológicos da Cl - possui 98 artigos, sendo 26 pesquisadores responsáveis por eles; o GT 4 - Gestão da Informação e do Conhecimento - possui 97 artigos, sendo 23 pesquisadores responsáveis por eles; o GT 5 - Política e Economia da Informação - possui 60 artigos, sendo 27 pesquisadores responsáveis por eles; o GT 10 - Informação e Memória - possui 52 artigos, sendo 14 pesquisadores responsáveis por eles; 0 GT 6 - Informação, Educação e Trabalho - possui 49 artigos, sendo 25 pesquisadores responsáveis por eles; o GT 3 - Mediação, Circulação e Apropriação da Informação - possui 48 artigos, sendo 16 pesquisadores responsáveis por eles; o GT 11 - Informação \& Saúde - possui 27 artigos, sendo 11 pesquisadores responsáveis por eles; o GT 9 - Museu, Patrimônio e Informação - possui dez artigos, sendo sete pesquisadores responsáveis por eles.

Nesse sentido, é possível apresentar algumas informações a partir de um olhar por pesquisador, considerando a dispersão dos pesquisadores por GT da ANCIB:

- Apenas uma pesquisadora publicou artigos agrupados em um só GT 
(Maria de F. G. M. Tálamo, GT 2).

- Nove pesquisadores tiveram seus trabalhos agrupados em dois GT:

Carlos C. de Almeida, GT 1 e 2; Ely F. T. de Oliveira, GT 2 e 7; Evelyn G. D. Orrico, GT 2 e 10; Gilda O de Oliveira, GT 4 e 5; José M. Jardim, GT 4 e 5; Maria L. de A. Campos, GT 2 e 8; Silvana D. Monteiro, GT 2 e 8; Sônia E. Caregnato, GT 7 e 8; e Vera L. D. L. de M. Dodebei, GT 2 e 10.

- Oito pesquisadores tiveram seus trabalhos agrupados em três GT, a saber: Carlos H. M. de Almeida, GT 2, 7 e 8; Edberto Ferneda, GT 2, 3 e 8; Marco A. F. Schneider, GT 1, 4 e 5; Maria C. C. Grácio, GT 2, 7 e 11; Maria C. P. I. Hayashi, GT 6, 7 e 11; Maria N. G. de Gomez, GT 1, 5 e 7; Rosângela S. Rodrigues, GT 6, 7 e 8; e Vinícius M. Kern, GT 1, 7 e 8.

- A produção de sete pesquisadores foi agrupada em quatro GT: Fernando César Lima Leite, GT 4, 5, 7 e 8; Gercina Â. de Lima, GT 2, 4, 6 e 7; Helen de C. S. Casarin, GT 4, 5, 6 e 7; Icleia Thiesen, GT 1, 3, 9 e 10; Marilda L. G. de Lara, GT 1, 2, 5 e 7; Nair Y. Kobashi, GT 2, 5, 8 e 10; e Renato R. Souza, GT 1, 2, 6 e 8.

- Agrupamos em cinco GT o trabalho de oito pesquisadores: Fábio A. Pinho, GT 1, 2, 4, 6 e 10; José A. C. Guimarães, GT 1, 2, 5, 7 e 8; Marco A. de Almeida, GT 1, 3, 5, 8 e 9; Maria A. Moura, GT 1, 2, 5, 6 e 8; Mariângela S. L. Fujita, GT 2, 3, 6, 7, 11; Regina M. Marteleto, GT 1, 2, 3, 4 e 11; Sámile A. de S. Vanz, GT 4, 6, 7, 8 e 11; e Sarita Albagli, GT 4, 5, 7, 8 e 11.

- Agrupamos em seis GT a produção de nove pesquisadores: Carlos A. Á. Araújo, GT 1, 3, 4, 6, 9 e 10; Emir J. Suaiden, GT 1, 4, 5, 6, 7 e 8; Gustavo S. Saldanha, GT 1, 2, 3, 5, 6 e 10; João B. E. de Moraes, GT 1, 2, 3, 5, 6 e 7; Lena V. R. Pinheiro, GT 1, 2, 5, 6, 7 e 10; Marisa B. B. Medeiros, GT 1, 2, 3, 6, 8 e 10; Silvana A. B. G. Vidotti, GT 2, 3, 4, 7, 8 e 11; Valdir J. Morigi, GT 3, 4, 5, 6, 9 e 10; e Virgínia B. Pinto, GT 2, 4, 5, 6, 8 e 11.

- Três pesquisadores foram agrupados em sete GT: Leilah S. Bufrem, GT 1, 2, 4, 5, 6, 7 e 11; Raimundo N. M. dos Santos, GT 2, 3, 4, 5, 6, 7 e 8; e Ricardo M. Pimenta, GT 1, 2, 3, 4, 5, 6 e 8.

- Agrupamos os artigos de cinco pesquisadores em oito GT: Adilson L. Pinto, GT 1, 2, 4, 5, 6, 7, 8 e 11; André P. A. Lopez, GT 1, 2, 4, 5, 7, 8, 9 e 10; 
Giulia Crippa, GT 1, 2, 3, 5, 6, 7, 9 e 10; Guilherme A. Dias, GT 1, 2, 4, 5, 6, 7, 8 e 10; e Marta L. P. Valentim, GT 1, 2, 3, 4, 5, 6, 7 e 8.

Ainda, um pesquisador teve trabalhos agrupados nos $11 \mathrm{GT}$ da ANCIB (Maurício B. Almeida).

Para Alves e Oliveira (2020, p. 139) os agrupamentos

[...] gerados por meio por meio dos indicadores de ligação entre os artigos publicados pelos pesquisadores de produtividade em pesquisa e os temas dos GTs da ANCIB, estão presentes características do campo (conceito que foi definido por Bourdieu combinando elementos presentes na Sociologia de Émile Durkheim, Karl Marx e Max Weber), e esses agrupamentos podem ser relacionados com essas características, considerando suas singularidades, estruturas sociais e propriedades.

Nesse sentido, a concentração de trabalhos em diferentes GTs da ANCIB pode significar a intenção dos pesquisadores em buscar a manutenção e conservação ou ocupar uma posição de destaque nessa estrutura social representada pelo Grafo 1. O conjunto de pesquisadores possuem interesses específicos que são definidos pelo campo e sua lógica (ALVES, 2018).

Apresenta-se o Gráfico 1, no qual se destaca a quantidade de GT em que os trabalhos foram classificados.

\section{Gráfico 1 - Quantidade de GT em que os trabalhos foram classificados}

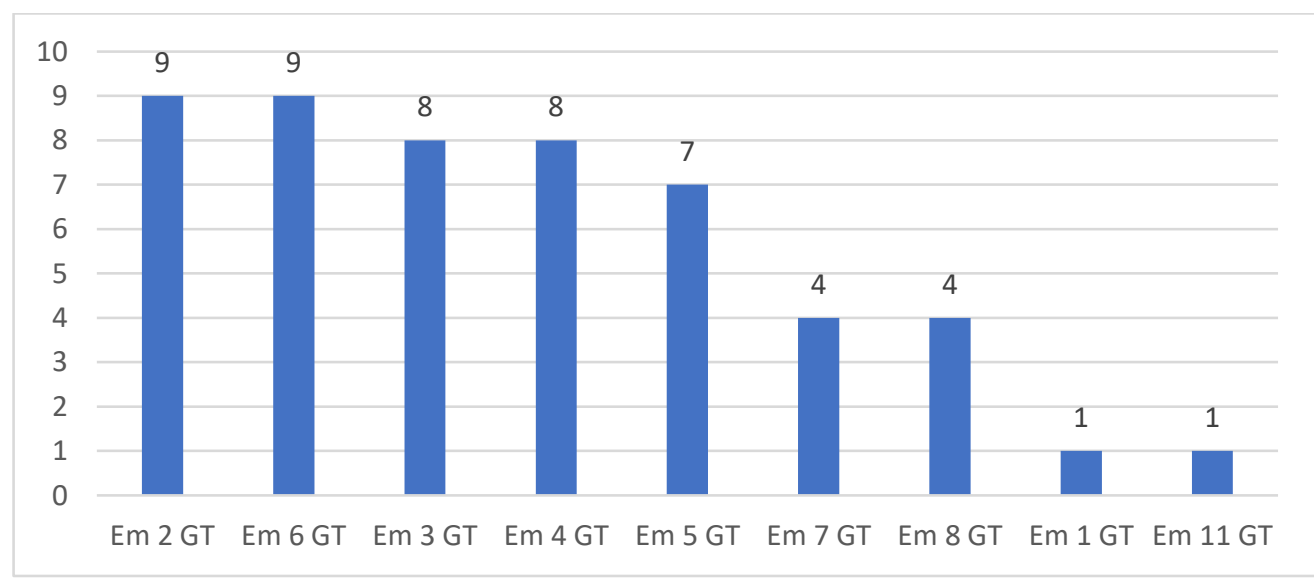

Fonte: Elaborado pelos autores.

A seguir, Tabela 2, apresentamos os pesquisadores mais produtivos em cada um deles: 
Tabela 2 - Pesquisadores mais produtivos em cada GT

\begin{tabular}{|c|c|c|c|c|c|}
\hline GT & Pesquisador & Quant.* $^{*}$ & GT & Pesquisador & Quant. \\
\hline \multirow[t]{3}{*}{ GT1 } & Gustavo S. Saldanha & 29 & \multirow[t]{3}{*}{ GT5 } & Emir J. Suaiden & 10 \\
\hline & Carlos A. Á. Araújo & 16 & & Valdir J. Morigi & 6 \\
\hline & Giulia Crippa & 9 & & Guilherme A. Dias & 4 \\
\hline \multirow[t]{3}{*}{ GT2 } & Mariângela S. L. Fujita & 32 & \multirow[t]{3}{*}{ GT7 } & Maria C. C. Grácio & 35 \\
\hline & José A. C. Guimarães & 30 & & Maria C. P. I. Hayashi & 32 \\
\hline & Gercina Â. de Lima & 24 & & Sámile A. de S. Vanz & 29 \\
\hline \multirow[t]{3}{*}{ GT3 } & Valdir J. Morigi & 7 & \multirow[t]{3}{*}{ GT8 } & Silvana A. B. G. Vidotti & 33 \\
\hline & Carlos A. Á. Araújo & 6 & & Guilherme A. Dias & 16 \\
\hline & Marco A. de Almeida & 6 & & Silvana D. Monteiro & 12 \\
\hline \multirow[t]{3}{*}{ GT4 } & Marta L. P. Valentim & 31 & GT9 & Giulia Crippa & 4 \\
\hline & Carlos A. Á. Araújo & 10 & \multirow[t]{3}{*}{ GT10 } & Evelyn G. D. Orrico & 10 \\
\hline & Helen de C. S. Casarin & 10 & & Giulia Crippa & 10 \\
\hline \multirow[t]{4}{*}{ GT6 } & Leilah S. Bufrem & 7 & & Dodebei & 9 \\
\hline & Carlos A. Á. Araújo & 4 & \multirow[t]{3}{*}{ GT11 } & Virginia B. Pinto & 7 \\
\hline & Maria A. Moura & 4 & & Regina M. Marteleto & 6 \\
\hline & Marta L. P. Valentim & 4 & & Maurício B. Almeida & 3 \\
\hline
\end{tabular}

Fonte: Elaborado pelos autores.

${ }^{*}$ Quantidade.

Isso não significa que o pesquisador publique majoritariamente na temática do GT, mas sim que teve alta produção ligada ao grupo. No caso do GT 9, Giulia Crippa é a única que publicou mais de um artigo. Os demais publicaram um artigo na temática e por isso não figuram da Tabela 2. Além disso, como veremos a seguir, apesar de ser a principal pesquisadora do tema na Tabela, Crippa de destaca por sua produção ligada ao GT 10.

No período sob análise, o GT7 e o GT2 apresentaram a maior concentração de trabalhos. Desse conjunto de pesquisadores representados pelos GTs acima, três (3) são docentes da Universidade Estadual Paulista "Júlio de Mesquita Filho" (UNESP-Marília); um (1) da Universidade Federal de São Carlos (UFSCar); um (1) da Universidade Federal do Rio Grande do Sul (UFRGS) e um (1) da Universidade Federal de Minas Gerais (UFMG).

Ainda, pode-se destacar que alguns dos pesquisadores representados pela Tabela 2 estão presentes em mais de um GT, por exemplo, Carlos A. Á. 
Araújo; Giulia Crippa, entre outros. Isso indica que os mesmos apresentam uma mobilidade entre os diferentes GTs da ANCIB, considerando os temas que são desenvolvidos por eles, objetos de estudos avaliados e os métodos de científicos empregados.

Esse conjunto de pesquisadores, Tabela 2, podem ser considerados a Elite de Pesquisa, tendo em vista o acúmulo do capital científico do tipo objetivado. Nesse sentido, essa espécie de capital pode ser representada pelos diferentes indicadores, por exemplo, indicador de produção (número de trabalhos publicados). (ALVES, 2018).

Por fim, esses aspectos são utilizados para avaliar o comportamento científico dos agentes inseridos em um determinado campo científico e também se estão contribuindo, de forma significativa, para a visibilidade e desenvolvimento da $\mathrm{Cl}$.

Ainda, Tabela 3, é possível ver qual a principal temática trabalhada por cada um dos 51 autores:

Tabela 3 - Temática trabalhada por cada pesquisador

\begin{tabular}{lcc}
\hline Pesquisador & GT principal & Artigos \\
\hline Adilson L. Pinto & GT 7 & 25 \\
André P. A. Lopez & GT 2 & 5 \\
Carlos A. Á. Araújo & GT 1 & 16 \\
Carlos C. de Almeida & GT 2 & 8 \\
Carlos H. M. de Almeida & GT 2, GT 8 & 7 em cada \\
Edberto Ferneda & GT 8 & 11 \\
Ely F. T. de Oliveira & GT 7 & 21 \\
Emir J. Suaiden & GT 5 & 10 \\
Evelyn G. D. Orrico & GT 10 & 10 \\
Fábio A. Pinho & GT 2 & 22 \\
Fernando César Lima Leite & GT 7 & 9 \\
Gercina Â. de Lima & GT 2 & 24 \\
Gilda O de Oliveira & GT 4 & 2 \\
Giulia Crippa & GT 10 & 10 \\
Guilherme A. Dias & GT 8 & 16 \\
Gustavo S. Saldanha & GT 1 & 29 \\
Helen de C. S. Casarin & GT 4 & 10 \\
Icleia Thiesen & GT 1 & 4 \\
João B. E. de Moraes & GT 2 & 5
\end{tabular}




\begin{tabular}{|c|c|c|}
\hline José A. C. Guimarães & GT 2 & 30 \\
\hline José M. Jardim & GT 4 & 2 \\
\hline Leilah S. Bufrem & GT 7 & 24 \\
\hline Lena V. R. Pinheiro & GT 7 & 4 \\
\hline Marco A. F. Schneider & GT 1 & 4 \\
\hline Marco A. de Almeida & GT 3 & 6 \\
\hline Maria A. Moura & GT 2 & 11 \\
\hline Maria C. C. Grácio & GT 7 & 35 \\
\hline Maria C. P. I. Hayashi & GT 7 & 32 \\
\hline Maria de F. G. M. Tálamo & GT 2 & 2 \\
\hline Maria L. de A. Campos & GT 2 & 6 \\
\hline Maria N. G. de Gomez & GT 1 & 3 \\
\hline Mariângela S. L. Fujita & GT 2 & 32 \\
\hline Marilda L. G. de Lara & GT 2 & 10 \\
\hline Marisa B. B. Medeiros & GT 2 & 16 \\
\hline Marta L. P. Valentim & GT 4 & 31 \\
\hline Maurício B. Almeida & GT 2 & 15 \\
\hline Nair Y. Kobashi & $\begin{array}{c}\text { GT 2, GT 5, GT 8, } \\
\text { GT } 10\end{array}$ & 1 em cada \\
\hline Raimundo N. M. dos Santos & GT 7 & 10 \\
\hline Regina M. Marteleto & GT 11 & 6 \\
\hline Renato R. Souza & GT 8 & 11 \\
\hline Ricardo M. Pimenta & GT 8 & 11 \\
\hline Rosângela S. Rodrigues & GT 7 & 20 \\
\hline Sámile A. de S. Vanz & GT 7 & 29 \\
\hline Sarita Albagli & GT 7 & 6 \\
\hline Silvana A. B. G. Vidotti & GT 8 & 33 \\
\hline Silvana D. Monteiro & GT 8 & 12 \\
\hline Sônia E. Caregnato & GT 7 & 24 \\
\hline Valdir J. Morigi & GT 10 & 8 \\
\hline Vera L. D. L. de M. Dodebei & GT 9 & 10 \\
\hline Vinícius M. Kern & GT 7, GT 8 & 2 em cada \\
\hline Virgínia B. Pinto & GT 11 & 7 \\
\hline
\end{tabular}

Fonte: Elaborado pelos autores.

Considerando a classificação dos artigos nos GT, teríamos que cinco pesquisadores têm sua produção principal ligada ao GT 1, 15 ao GT 2, um ao GT 3, quatro no GT 4, dois no GT 5, nenhum no GT 6, 13 no GT 7, nove no GT 8, um no GT 9, quatro no GT 10, e 2 no GT 11.

Isso indica, que os pesquisadores podem adotar estratégias sociais com 
objetivo de contribuir para o desenvolvimento da temática do GT e/ou em busca de visibilidade junto aos seus pares, ou seja, acúmulo de capital científico do tipo puro. Nesse sentido, essa inserção cientifica ocorre, principalmente, por meio das descobertas científicas que podem ser utilizadas para repensar determinada estrutura social e/ou científica. Com isso, pode ocorrer a ruptura de paradigmas em um determinado campo científico. (ALVES, 2018).

Apresenta-se, Gráfico 2, com a quantidade de pesquisadores por GT da ANCIB.

\section{Gráfico 2 - Quantidade de pesquisadores por GT}

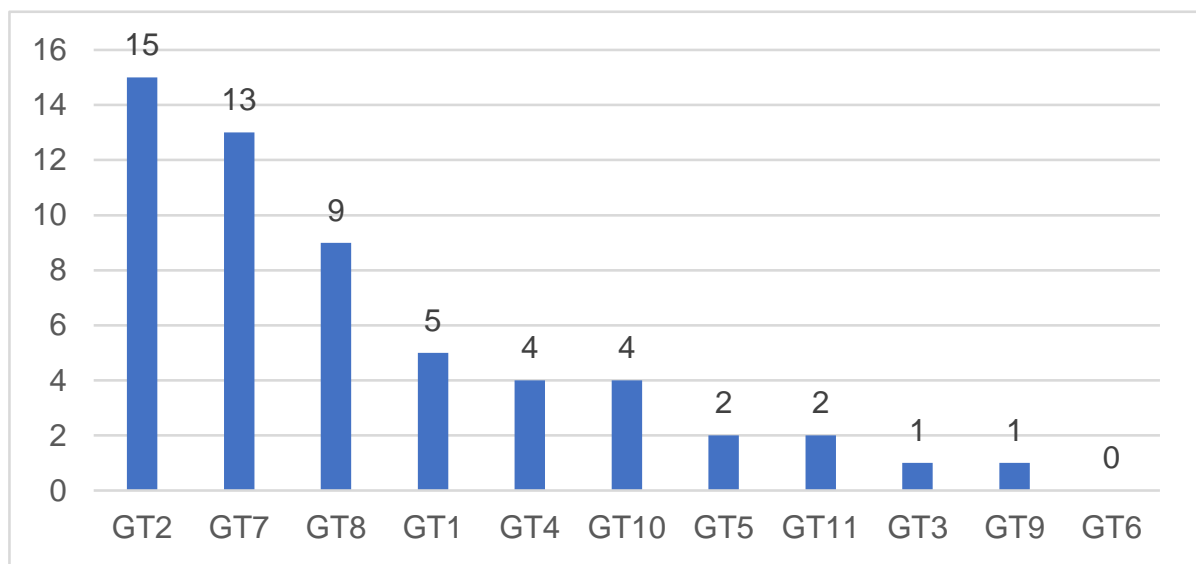

Fonte: Elaborada pelos autores.

Considerando o número de artigos no GT em que mais se destacou em relação ao total de classificações neste estudo, podemos organizar os pesquisadores pelo nível de aderência ao GT, sendo aderência muito baixa de 1 a $20 \%$, aderência baixa, de 21 a $40 \%$, aderência média, de 41 a $60 \%$, aderência alta, de 61 a $80 \%$, e aderência muito alta de 81 a 100\%. Encontramos o seguinte resultado: 13 pesquisadores apresentam aderência muito alta, 15 alta, 16 média, sete baixa, e nenhum apresentou aderência muito baixa.

\section{CONSIDERAÇÕES FINAIS}

Buscou-se desenvolver uma análise sistematizada baseada nos resultados para responder a seguinte questão: como os artigos produzidos pelos pesquisadores de produtividade em pesquisa em $\mathrm{Cl}$ contribuem para a construção do conhecimento no Campo em estudo? Como é o comportamento 
de publicação de nossos pesquisadores?

O conhecimento científico gerado pelos pesquisadores de produtividade em pesquisa em $\mathrm{Cl}$ são representados, por exemplo, por meio dos artigos científicos. Com isso, eles podem contribuir de forma significativa para o desenvolvimento do campo científico, por exemplo, esse conhecimento foi gerado a partir de uma estrutura sistematizada com o objetivo de atender as necessidades de um espaço social que apresenta relações objetivas entre os diferentes agentes e também instituições. Nesse sentido, é necessário a divulgação e disseminação desse conhecimento científico por meio dos diferentes veículos de comunicação.

Nesse contexto, o campo científico terá acesso as informações, considerando os objetos de estudos e problemas que foram investigados em determinado período, tendo em vista os métodos científicos empregados. A partir dessas características é possível pensar em uma tomada de decisão coerente com a realidade do agente e também da instituição.

Ainda, a dispersão dos artigos pode ocorrer por conta das ementas dos GT, que eventualmente, propõem descrições que acabam esbarrando em outro GT, como, por exemplo, é o caso de GT 2 e GT 8. Ambos discutem a questão da organização do conhecimento, sendo que a questão tecnológica é obrigatória ao GT 8, mas pode ser apresentada no GT 2.

Ressaltamos que uma proposta de agrupamento dessa natureza é arbitrária e pode (e deve ser) aprimorada em estudos futuros. Não é objetivo desta pesquisa analisar 0 alcance das ementas, cabe ressaltar essas características, que muitas vezes nos impediu de classificar um artigo dentro de um único GT. Possivelmente essa dificuldade apresenta-se aos pesquisadores que desejam submeter uma comunicação ao ENANCIB, e deixamos a sugestão de uma revisão das ementas de modo a facilitar a ação de submissão.

Vimos também trabalhos que escapam do escopo da $\mathrm{Cl}$, ou melhor dizendo, escapam do escopo das ementas dos 11 GT da ANCIB. Possivelmente, o fato pode ser explicado por pesquisadores com atuação em mais de uma área de saber como: Comunicação, Saúde, Ciências Agrárias, biológicas, entre outras. 
Como avaliar a aderência do pesquisador ou a dispersão de seus interesses de pesquisa? Se por um lado, ater-se a uma temática pode levar a um alto nível de especialidade, por outro lado, um movimento horizontal entre as temáticas abre possibilidade de pesquisa e pode agregar novos interesses e estimular a criatividade. Vale ressaltar, de todo modo, que as bolsas $P Q$ são distribuídas a pesquisadores que tenham um projeto de pesquisa, e de certa forma, espera-se que sua produção reflita tais temas.

Destacamos o número de pesquisadores que desenvolvem pesquisas relacionadas as atividades dos GT 2, 7 e 8, por um lado, e a ausência de pesquisadores produtivos nas temáticas relacionadas ao GT 6, que aborda justamente a formação e o perfil esperado nas áreas da $\mathrm{Cl}$. Com o cenário apresentado, arriscaríamos afirmar que os pesquisadores de produtividade em pesquisa em $\mathrm{Cl}$ abordam questões relacionadas à organização, recuperação e produção do conhecimento. Recuperando Bourdieu, talvez estas áreas recompensem mais seus pesquisadores, em termos de renome, e certamente na quantidade de bolsas oferecidas.

Como recomendações para o prosseguimento desta pesquisa, destacase a relevância de se identificar o capital agregado e também aqueles pesquisadores de produtividade em pesquisa em $\mathrm{Cl}$ que possuem artigos indexados nas diferentes bases de dados internacionais. Esses indicadores podem ser utilizados para identificar os dominantes, dominados e pretendentes em um determinado campo científico.

\section{REFERÊNCIAS}

ALVES, B. H.; OLIVEIRA, E. F. T. de. Sociologia de Bourdieu: avaliação da produção científica dos pesquisadores de produtividade em pesquisa em Ciência da Informação. Em Questão, Porto Alegre, v. 26, n. 3, p. 121-145, set./dez. 2020. Disponível em:

https://seer.ufrgs.br/EmQuestao/article/view/93092/56607. Acesso em: 23 set. 2020.

ALVES, B. H. Sociologia de Pierre Bourdieu e os pesquisadores bolsistas de produtividade em pesquisa do CNPq em Ciência da Informação. 2018. Tese (Doutorado em Ciência da Informação) - Faculdade de Filosofia e Ciências, Universidade Estadual Paulista, Marília, 2018. 
ARAÚJO, R. F.; ALVES, M. Altmetria e atenção online da produção de pesquisadores brasileiros: análise dos bolsistas de produtividade do cnpq. In: ENCONTRO BRASILEIRO DE BIBLIOMETRIA E CIENTOMETRIA, 6., 2018, Rio de Janeiro. Anais [...]. Rio de Janeiro: UFRJ, 2018. p. 234-242.

ARAÚJO, R. F.; ALVES, M. Indicadores altmétricos da produção de pesquisadores brasileiros: análise dos bolsistas de produtividade do cnpq. Revista Ibero-Americana de Ciência da Informação, Brasília, v. 12, n. 3, p. 850-862, set./dez. 2019. DOI: 10.26512/rici.v12.n3.2019.17203. Disponível em:https://www.researchgate.net/publication/335878896_Indicadores_altmetric os_da_producao_de_pesquisadores_brasileiros_analise_dos_bolsistas_de_pro dutividade_do_CNPPq. Acesso em: 21 mar. 2020.

ASSOCIAÇÃO NACIONAL DE PESQUISA E PÓS-GRADUAÇÃO EM CIÊNCIA DA INFORMAÇÃO [ANCIB]. Sobre. 2016. Disponível em: http://www.ancib.org.br/. Acesso em: 1 mar. 2017.

BOURDIEU, P. Os usos sociais da ciência: por uma sociologia clínica do campo científico. São Paulo: Editora Unesp, 2004.

BOURDIEU, P. Para uma sociologia da ciência. Lisboa: Ed. 70, 2008.

BUFREM, L. S.; OLIVEIRA, E. F. T. de; SOBRAL, N. V.; ALVES, B. H. Temas relacionados à educação na produção científica periódica dos bolsistas de produtividade em pesquisa da área de ciência da informação no brasil. Bibliotecas. Anales de Investigación, Cuba, v. 14, n. 2, p. 179-192, jul. 2018. Disponível em: http://eprints.rclis.org/33186/. Acesso em: 21 mar. 2020.

BUFREM, L. S.; OLIVEIRA, E. F. T. de; SOBRAL, N. V. Produção científica sobre temas pertinentes ao gt 07 indexada na base de dados brapci. In: ENCONTRO NACIONAL DE PESQUISA EM CIÊNCIA DA INFORMAÇÃO, 19., 2018, Londrina. Anais [...]. Londrina: UEL, 2018. p. 4292-4608.

BUFREM, L. S.; SILVA, F. M. E.; SOBRAL, N. V. Análise das influências intelectuais na produção científica da área de ciência da informação: um estudo sobre os bolsistas de produtividade em pesquisa (pq-cnpq). Em Questão, Porto Alegre, v. 23, p. 115-141, Edição Especial 5 EBBC, 2017.

DOI: 10.19132/1808-5245230.115-141. Disponível em: https://seer.ufrgs.br/EmQuestao/article/view/68087. Acesso em: 21 mar. 2020.

CAFÉ, A. L. da P.; CARVALHO, K.; MENEZES, V.; ODDONE, N. A elite acadêmica da sociologia no brasil e sua produção científica. Informação \& Informação, Londrina, v. 16, n. 3, p. 19-39, jan./jun. 2011. DOI: 10.5433/19818920.2011v16n3p19. Disponível em:

http://www.uel.br/revistas/uel/index.php/informacao/article/view/9967. Acesso em: 21 mar. 2020.

CARVALHO, K.; ODDONE, N.; CAFÉ, A. L. da P.; MENEZES, V. Aspectos gerenciais da política científica brasileira: um olhar sobre a produção científica 
do campo da sociologia face aos critérios de avaliação do CNPq e da CAPES. Em Questão, Porto Alegre, v. 19, n. 1, p. 187-212, jan./jun. 2013. Disponível em: https://seer.ufrgs.br/EmQuestao/article/view/36908. Acesso em: 21 mar. 2020.

CASTANHA, R. G.; GRÁCIO, M. C. C. Autoria na produção científica da ciência da informação: uma análise dos artigos dos bolsistas produtividade em pesquisa (2012-2016). In: ENCONTRO NACIONAL DE PESQUISA EM CIÊNCIA DA INFORMAÇÃO, 18., 2017, Marília. Anais [...]. Marília: UNESP, 2017.

CASTANHA, R. G.; VALENTIM, M. L. P. O perfil disciplinar da ciência da informação no brasil: um estudo em torno da formação disciplinar dos bolsistas de produtividade em pesquisa do cnpq. Pesquisa Brasileira em Ciência da Informação e Biblioteconomia, João Pessoa, v. 13, n. 1, p. 010-020, 2018. DOI: 10.22478/ufpb.1981-0695.2018v13n1.39469. Disponível em: https://www.researchgate.net/publication/332575202_O_perfil_disciplinar_da_C iencia_da_Informacao_no_Brasil_um_estudo_em_torno_da_formacao_disciplin ar_dos_bolsistas_de_produtividade_em_pesquisa_do_CNPq. Acesso em: 21 mar. 2020.

CONSELHO NACIONAL DE DESENVOLVIMENTO CIENTÍFICO E TECNOLÓGICO [CNPq]. Bolsas Individuais no país. RN-028/2015. Brasília, 2015. Publicada no DOU de 21/12/15, Seção 1, pág. 45. Disponível em: http://cnpq.br/view//journal_content/56_INSTANCE_0oED/10157/2958271?COMPANY_ID=10132 \#PQ . Acesso em: 21 mar. 2020.

CRUZ, T. L.; SILVA, F. M. E.; BUFREM, L. S.; SOBRAL, N. V. Influências intelectuais dos bolsistas de produtividade em ciência da informação no brasil (2008 a 2017). In: ENCONTRO BRASILEIRO DE BIBLIOMETRIA E CIENTOMETRIA, 6., 2018, Rio de Janeiro. Anais [...]. Rio de Janeiro: UFRJ, 2018. p. 1-14.

CRUZ, T. L.; SILVA, F. M. E.; BUFREM, L. S. Bolsistas de produtividade em ciência da informação no brasil e aspectos de suas influências intelectuais. In: ENCONTRO NACIONAL DE PESQUISA EM CIÊNCIA DA INFORMAÇÃO, 19., 2018, Londrina. Anais [...]. Londrina: UEL, 2018. p. 4732 - 4740.

DARAIO, C. VACCARI, A. Using normative ethics for building a good evaluation of research practices: towards the assessment of researcher's virtues.

Scientometrics, Netherlands, 17 Aug, 2020. Disponível em: https://doi.org/10.1007/s11192-020-03658-4. Acesso em: 19 set. 2020

DROESCHER, F. D.; SILVA, E. D. da. O pesquisador e a produção científica. Perspectivas em Ciência da Informação, Belo Horizonte, v. 19, n. 1, p. 170 189, jan./mar. 2014. Disponível em: 
https://www.scielo.br/scielo.php?pid=S1413-

99362014000100011\&script=sci_abstract\&tIng=pt. Acesso em: 23 set. 2020.

ENCONTRO NACIONAL DE PESQUISA EM CIÊNCIA DA INFORMAÇÃO [ENANCIB]. Grupos de Trabalhos (GTs). 2019. Disponível em:

http://www.enancib2019.ufsc.br/. Acesso em: 21 mar. 2020.

FERREIRA, T. G.; CASARIN, H. C. S.; MATTOS, N. B. Habilidades de busca de pesquisadores brasileiros no domínio da educação. Brazilian Journal of Information Science, Marilia, v. 1, n. 3, 2016. DOI: 10.5016/brajis.v10i3.6448. Disponível em:

http://www2.marilia.unesp.br/revistas/index.php/bjis/article/view/6448. Acesso em: 21 mar. 2020.

GARVEY, W. D. Communication: the essence of science; facilitating information among librarians, scientists, engineers and students. Oxford: Pergamon, 1979.

GARVEY, W. D., GRIFFITH, B. C. Communication and information process within scientific disciplines, empirical findings for psychology. In: GARVEY, W. D. Communication: the essence of science; facilitating information among librarians, scientists, engineers and students. Oxford: Pergamon, 1979. p. 127147.

GIDDENS, A. A Constituição da Sociedade. São Paulo: Martins Fontes, 1989.

GUIMARÃES, J. A. C.; GRÁCIO, M. C. C.; MATOS, D. F. O. Produção científica de bolsistas pesquisa em ciência da informação do conselho nacional de desenvolvimento científico e tecnológico (cnpq) - um estudo com artigos de periódicos. DataGramaZero, v. 15, n. 2, abr. 2014. Disponível em: http://hdl.handle.net/20.500.11959/brapci/8279. Acesso em: 22 jan. 2020.

HANNEMAN, R. A.; RIDDLE, M. Introduction to social network methods. Riverside: University of Califórnia, 2005.

HARGENS, L. L. What is Mertonian sociology of science? Scientometrics, Netherlands, v. 60, n. 1, p. 63-70, 2004. Disponível em: https://link.springer.com/article/10.1023/B:SCIE.0000027309.30756.6c . Acesso em: 18 set. 2020.

LE COADIC, Y.-F. A ciência da informação. Brasília: Briquet de Lemos, 2004.

LEYDESDORFF, L. Scientific communication and cognitive codification: social systems theory and the sociology of scientific knowledge. European Journal of Social Theory, v. 10, i. 3, p. 1-22, 2007. Disponível em: https://doi.org/10.1177/1368431007080701. Acesso em: 19 set. 2020. 
MEADOWS, A. J. A comunicação científica. Brasília: Briquet de Lemos, 1999.

MELO, W. L.; BUFREM, L. S.; CORREIA, A. E. G. C. Bolsistas de produtividade em pesquisa 1 em ciência da informação: verificação da produtividade e percepções autorais em um ambiente de comunicação científica. In: ENCONTRO NACIONAL DE PESQUISA EM CIÊNCIA DA INFORMAÇÃO, 19., 2018, Londrina. Anais [...]. Londrina: UEL, 2018. p. 43604378.

MELO, W. L.; CORREIA, A. E. G. C. Indicativos do campo científico: análise da produtividade dos bolsistas pq 1 e pq 2 em comunicação (2004-2013). Em Questão, Porto Alegre, v. 23, p. 9-30, Edição Especial 5 EBBC, 2017. DOI: 10.19132/1808-5245230.9-30. Disponível em: https://seer.ufrgs.br/EmQuestao/article/view/68037/40132.Acesso em: 21 mar. 2020.

MENEZES, V. S.; ODDONE, N. E.; CAFÉ, A. L. P. Aspectos reputacionais dos sistemas de avaliação da produção científica no campo da ciência da informação. Tendências da Pesquisa Brasileira em Ciência da Informação, v. 5, n. 1, 2012. Disponível em:

http://hdl.handle.net/20.500.11959/brapci/119440. Acesso em: 22 jan. 2020.

MERTON, R. K. The sociology of science: theoretical and empirical investigations. Chicago: The University of Chicago, 1973.

MOODY, J. The structure of a social science collaboration network: Disciplinary cohesion from 1963 to 1999. American Sociological Review, v. 69, p. 213238, April 2004. Disponível em:

https://www.soc.duke.edu/ jmoody77/asr_soccoauth.pdf .Acesso em: 19 set. 2020.

OLIVEIRA, C. A.; OLIVEIRA, M.; DIAS, T. M. R.; COSTA, B. I. R. Genealogia acadêmica dos pesquisadores da área de ciência da informação: um estudo sobre os bolsistas de produtividade em pesquisa (pq-cnpq). Em Questão, Porto Alegre, v. 24, p. 278-298, 2018. DOI: 10.19132/1808-5245240.278-298. Disponível https://seer.ufrgs.br/EmQuestao/article/view/86929/52349. Acesso em: 21 mar. 2020.

OLIVEIRA, C. A.; COSTA, B. I. R.; OLIVEIRA, M. Genealogia acadêmica: um estudo dos pesquisadores da área de ciência da informação com bolsas produtividade em pesquisa na modalidade pq-1 do cnpq. In: ENCONTRO BRASILEIRO DE BIBLIOMETRIA E CIENTOMETRIA, 6., 2018, Rio de Janeiro. Anais [...]. Rio de Janeiro: UFRJ, 2018. p. 291-300.

OLIVEIRA, E. F. T.; GRACIO, M. C. C. A produção científica em organização e representação do conhecimento no Brasil: uma análise bibliométrica do GT-2 da ANCIB. In: ENCONTRO NACIONAL DE PESQUISA EM CIÊNCIA DA 
INFORMAÇÃO, 10., 2009, João Pessoa. Anais [...]. João Pessoa: UFPB, 2009. p. 1-19.

PEREIRA, C. A. Cartografia dos Estudos Métricos da Informação: panorama atual, desafios e perspectivas na avaliação da ciência. 2015. Tese (Doutorado em Ciência da Informação) - Escola de Comunicações e Artes, Universidade de São Paulo, São Paulo, 2015.

PRICE, D. S. A ciência desde a Babilônia. Belo Horizonte: Itatiaia, 1976.

SCOTT, W. R. Institutions and organizations. 2. ed. Thousand Oaks: Sage, 2001.

SMIT, J. W.; BARRETO, A. A. A Ciência da Informação: base conceitual para a formação do profissional. In: VALENTIM, M. L. (Org.). Formação do profissional da informação. São Paulo: Polis, 2002. p. 9-23.

TARGINO, M. G. Comunicação científica: uma revisão de seus elementos básicos. Informação \& Sociedade: Estudos, João Pessoa, v. 10, n. 2, jan. 2000. Disponível em:

https://periodicos.ufpb.br/ojs/index.php/ies/article/view/326/248. Acesso em: 21 mar. 2020.

VAN RAAN, A. F. J. The use of bibliometric analysis in research performance assessment and monitoring of interdisciplinary scientific developments.

Technikfolgenabschätzung - Theorie und Praxis, Karlsruhe, v. 1, n. 12, p. 20-29, 2003. Disponível em:

http://citeseerx.ist.psu.edu/viewdoc/download?doi=10.1.1.153.629\&rep=rep1\&ty pe=pdf. Acesso em: 21 mar. 2020.

VESSURI, H. M. C. The Social Study of Science in Latin America. Social Studies of Science, Thousand Oaks, v. 17, n. 3, p. 519-554, aug. 1987.

Disponível em: https://www.jstor.org/stable/285135?seq=1. Acesso em: 21 mar. 2020.

WAGNER, C. S.; LEYDESDORFF, L. Network structure, self-organization, and the growth of international collaboration in science. Research Policy, v. 34, p. 1608-1618, 2005. Disponível em: https://arxiv.org/abs/0911.4299 . Acesso em 19 set. 2020.

ZIMAN, J. M. Real science: what it is, and what it means. Cambrige; New York: Cambridge University Press, 2000.

\section{THE PRODUCTION OF CNPq PRODUCTIVITY SCHOLARSHIPS IN INFORMATION SCIENCE AND ITS RELATION TO ENANCIB THEMES}




\begin{abstract}
Introduction: Sociology of Science seeks to understand the behavior of researchers and offer subsidies for understanding their publishing practices. When considering the National Research Meeting in Information Science (ENANCIB) as the main conference in Information Science (IS) in Brazil, verifying the publication of productivity fellows of the National Council for Scientific and Technological Development (CNPq) in the light of the Working Groups (WG) of the event allows us to establish a scenario of the most popular IS themes worked by these researchers. Objective: The research aims to understand the construction of scientific knowledge published by CNPq productivity fellows, grouping their articles according to the WG of the National Association of Research and PostGraduation in Information Science (ANCIB). Methodology: This is a bibliographic research of quantitative, qualitative and exploratory nature, supported by bibliometrics. Results: The 51 fellows published 883 articles. The articles were grouped mainly in WG7, 2 and 8, and the WG1 0 with the fewest articles. The most productive researchers by WG were identified, in addition to their themes and the level of researchers' adherence to the theme. Conclusions: We highlight the number of researchers developing research related to the activities of WG 2, 7 and 8 , and the absence of productive researchers in the themes related to WG6, which precisely addresses training and expected profile in IS. With the presented scenario, we would risk to state that productivity fellows address issues related to the knowledge organization, retrieval and production.
\end{abstract}

Descriptors: CNPq Productivity Fellows. Research themes. Scientific Communication. ANCIB WG.

\title{
PRODUCCIÓN DE LOS BECARIOS DE PRODUCTIVIDAD CNPq EN LA CIENCIA DE LA INFORMACIÓN Y SU RELACIÓN CON TEMAS ENANCIB
}

\begin{abstract}
RESUMEN
Introducción: La Sociología de la Ciencia intenta comprender el comportamiento de los investigadores y ofrecer subsidios para comprender sus prácticas editoriales. Al considerar la Reunión Nacional de Investigación en Ciencia de la Información (ENANCIB) como conferencia principal en la Ciencia de la Información (Cl) en Brasil, verificar la publicación de becarios de productividad del Consejo Nacional para el Desarrollo Científico y Tecnológico (CNPq) a la luz de los Grupos de Trabajo (GT) del evento permite establecer un escenario de los temas más populares de estos investigadores. Objetivo: La investigación objetiva comprender la construcción del conocimiento científico publicado por los becarios del CNPq, agrupando sus artículos de acuerdo con los GT de la Asociación Nacional de Investigación y Postgrado en Ciencias de la Información (ANCIB). Metodología: Investigación bibliográfica de naturaleza cuantitativa, cualitativa y exploratoria, respaldada por bibliometría. Resultados: Los 51 becarios publicaron 883 artículos. Los artículos se agruparon principalmente en los GT7, 2 y 8, y el GT10 con la menor cantidad de artículos. Se identificaron los investigadores más productivos de los GT, sus temas y el nivel de adhesión de los investigadores al tema. Conclusiones: Destacamos el número de investigadores que desarrollan investigaciones relacionadas con los GT2, 7 y 8, y la ausencia de investigadores productivos en los temas del GT6, que aborda precisamente la capacitación y el perfil esperado en la Cl. Con el escenario presentado, correríamos
\end{abstract}


el riesgo de afirmar que los becarios de productividad abordan cuestiones relacionadas con la organización, recuperación y producción del conocimiento

Descriptores: Becarios de Productividad CNPq. Temas de investigación. Comunicación científica. GT de la ANCIB.

Recebido em: 26.10.2020

Aceito em: 05.02.2021 